This item was submitted to Loughborough's Research Repository by the author.

Items in Figshare are protected by copyright, with all rights reserved, unless otherwise indicated.

\title{
Geometric stochastic resonance
}

PLEASE CITE THE PUBLISHED VERSION

http://dx.doi.org/10.1103/PhysRevLett.104.020601

PUBLISHER

(C) American Physical Society

VERSION

VoR (Version of Record)

LICENCE

CC BY-NC-ND 4.0

REPOSITORY RECORD

Ghosh, Pulak Kumar, Fabio Marchesoni, Sergey Savel'ev, and Franco Nori. 2019. "Geometric Stochastic Resonance". figshare. https://hdl.handle.net/2134/12717. 
This item was submitted to Loughborough's Institutional Repository (https://dspace.lboro.ac.uk/) by the author and is made available under the following Creative Commons Licence conditions.

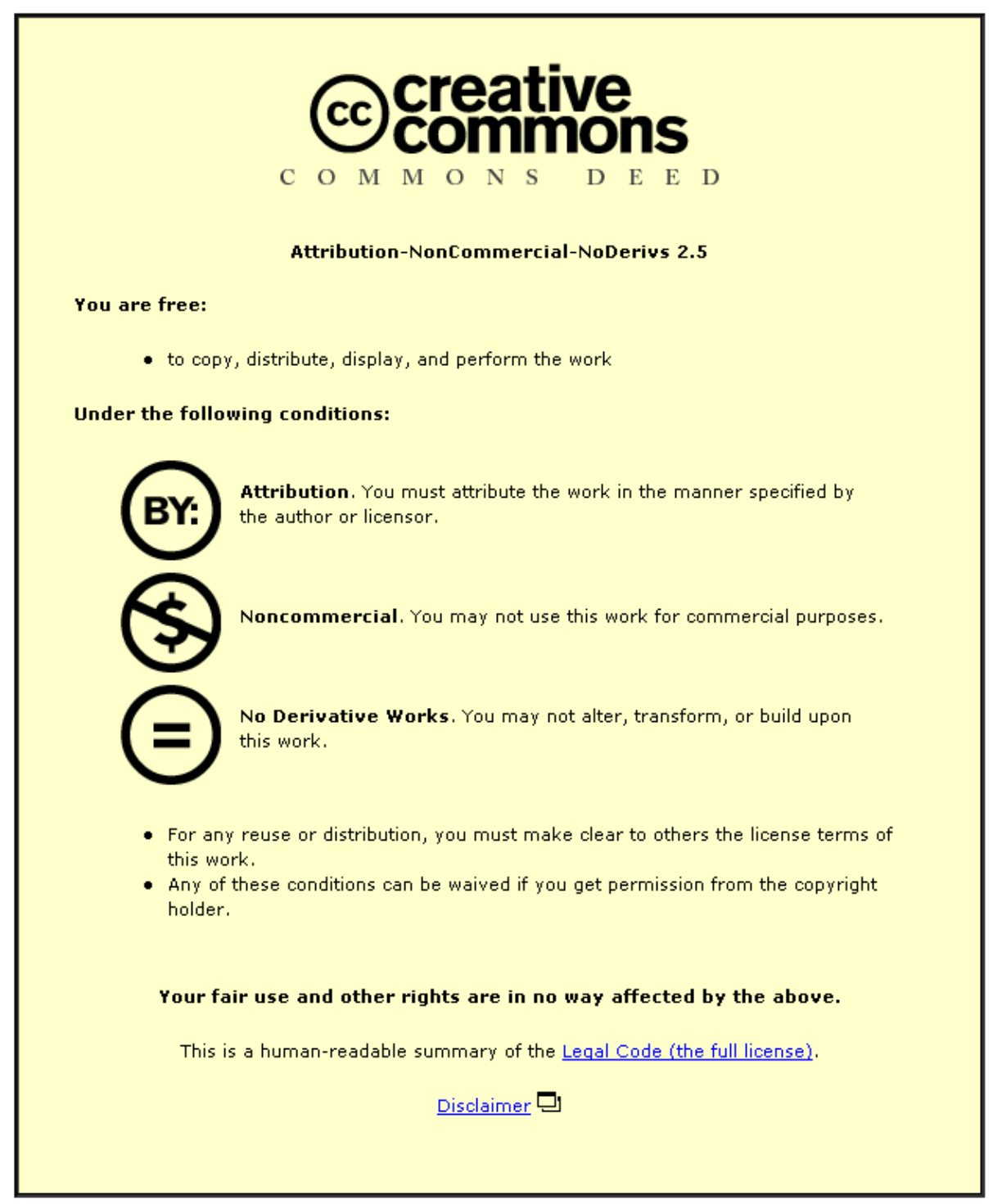

For the full text of this licence, please go to: http://creativecommons.org/licenses/by-nc-nd/2.5/ 


\title{
Geometric Stochastic Resonance
}

\author{
Pulak Kumar Ghosh, ${ }^{1}$ Fabio Marchesoni, ${ }^{2,1}$ Sergey E. Savel'ev, ${ }^{3,1}$ and Franco Nori ${ }^{1,4}$ \\ ${ }^{1}$ Advanced Science Institute, The Institute of Physical and Chemical Research (RIKEN), Wako-shi, Saitama, 351-0198, Japan \\ ${ }^{2}$ Dipartimento di Fisica, Università di Camerino, I-62032 Camerino, Italy \\ ${ }^{3}$ Department of Physics, Loughborough University, Loughborough LE11 3TU, United Kingdom \\ ${ }^{4}$ Department of Physics, University of Michigan, Ann Arbor, Michigan 48109-1040, USA
}

(Received 17 July 2009; published 14 January 2010)

\begin{abstract}
A Brownian particle moving across a porous membrane subject to an oscillating force exhibits stochastic resonance with properties which strongly depend on the geometry of the confining cavities on the two sides of the membrane. Such a manifestation of stochastic resonance requires neither energetic nor entropic barriers, and can thus be regarded as a purely geometric effect. The magnitude of this effect is sensitive to the geometry of both the cavities and the pores, thus leading to distinctive optimal synchronization conditions.
\end{abstract}

DOI: 10.1103/PhysRevLett.104.020601

PACS numbers: 05.40.- a, 05.10.Gg

Stochastic resonance (SR) is a textbook example of how noise can best enhance the response of a bistable system to an external drive [1]. Historically, research on SR focused mostly on systems with purely energetic potentials, either continuous or discrete. However, as pointed out in Ref. [2], in soft condensed matter and in a variety of biological systems [3], particles are often confined to constrained geometries, such as interstices, pores, or channels, whose size and shape can affect the SR mechanism [4]. Indeed, smooth confining geometries can be modeled as entropic (i.e., noise or temperature dependent) potentials [5], capable of influencing the response of the system to an external driving force [6].

Let us consider a Brownian particle freely diffusing in a two (2D) or three dimensional suspension fluid contained in two symmetric cavities connected by a narrow pore; it can switch cavity only by overcoming the entropic barrier determined by the geometric constriction associated with the pore. This is true even for ideal reflecting boundaries, that is, in the absence of an intrinsic energetic barrier. Burada et al. [4] have shown that entropic barriers significantly contribute to the magnitude of the SR effect that occurs when a periodic force drives the particle across the pore, hence the term of "entropic SR" coined in Ref. [4]. The evidence reported there, however, hints at an interplay of entropic and energetic barriers, rather than to a mere entropic effect. Indeed, SR was demonstrated there only under the explicit condition that the applied field of force had a dc component at an angle with the pore axis, say orthogonal to it, so that the particle tended to sojourn preferably against one side of the cavities. In the absence of such an additional symmetry-breaking force, no SR was observed.

This remark raises the issue whether a bistable effective potential is a necessary condition for SR to occur altogether [7]. In this Letter we show that a Brownian particle confined to two distinct cavities divided by a porous me- dium, say a membrane, does undergo SR when driven by an ac force perpendicular to the membrane, even in the absence of external gradients and/or interactions with the walls (besides bouncing from the walls). At variance with ordinary SR, optimal synchronization between drive and particle oscillations for an appropriate noise level, only occurs at driving frequencies (amplitudes) lower (higher) than a certain onset threshold. Moreover, such a manifestation of SR in higher dimensions requires adopting extremely sharp geometrical constrictions to separate the two cavities, something akin to the pores obtained by puncturing a thin membrane. The magnitude and conditions of the effect reported here are sensitive to both the geometry of the cavities and the cross section of the pores, thus allowing a direct control of the synchronization mechanism.

The overdamped dynamics of a Brownian particle in 2D is modeled by the Langevin equation

$$
d \vec{r} / d t=-A(t) \vec{e}_{x}+\sqrt{D} \vec{\xi}(t)
$$

where $\vec{e}_{x}, \vec{e}_{y}$ are the unit vectors along the $x, y$ axes and $\vec{\xi}(t)=\left(\xi_{x}(t), \xi_{y}(t)\right)$ are zero mean, white Gaussian noises with autocorrelation functions $\left\langle\xi_{i}(t) \xi_{j}\left(t^{\prime}\right)\right\rangle=2 \delta_{i j} \delta\left(t-t^{\prime}\right)$ with $i, j=x, y$. Equation (1) was numerically integrated by using a Milstein algorithm [8] for the two-cavity container sketched in Fig. 1, with reflecting walls [9] and a single opening, with different geometries, placed at the center of the partition wall. Stochastic averages were obtained as ensemble averages over $10^{6}$ trajectories with random initial conditions; transient effects were estimated and subtracted. In the presence of an ac drive, $A(t)=$ $A_{0} \cos (\Omega t)$, the Brownian trajectories embed a persistent harmonic component, $\bar{x}(D) \cos [\Omega t-\phi(D)]$, whose amplitude, $\bar{x}$, and phase, $\phi$, have been numerically fitted from data, and plotted versus $D$ in Figs. 2-4.

For simplicity, we start modeling the pore as a structureless hole pierced in a zero-thickness wall. The occurrence of a SR phenomenon is clear, albeit with some distinctive 

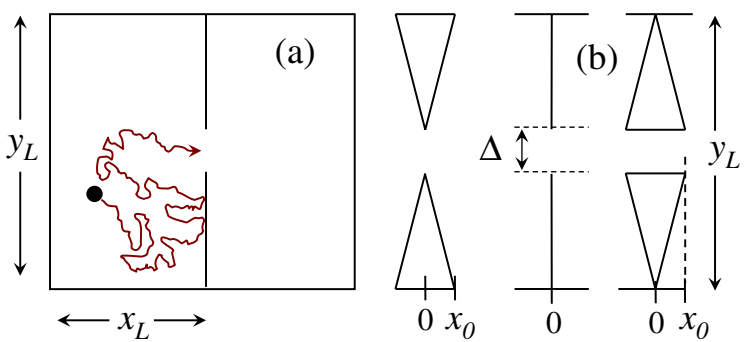

FIG. 1 (color online). (a) Brownian particle confined to a 2D box divided in two compartments by a partition with an opening at the center. (b) Three different pore geometries are used in our simulations: funnel, hole, and spout (left to right). $\Delta$ and $x_{0}$ denote, respectively, the cross section and the thickness of the pore.

features, as seen in Figs. 2 and 3: (i) $\bar{x}(D)$ peaks for an appropriate noise intensity, $D_{\max }$, with $D_{\max }$ a manifestly increasing function of $\Omega$; (ii) SR is restricted to $A_{0}>A_{c}$, Fig. 2(a), and $\Omega<\Omega_{c}$, Fig. 2(b). This is an important difference with respect to ordinary SR, where there exist no such onset thresholds in the drive parameter space; (iii) the curvres $\bar{x}(D)$ decay like $D^{-1}$, Fig. 3 , that is faster than in any 1D bistable potential and more in line with SR in a discrete two-state model [10].

All properties listed above can be explained by simple geometrical considerations. When the ac force $A(t)$ presses
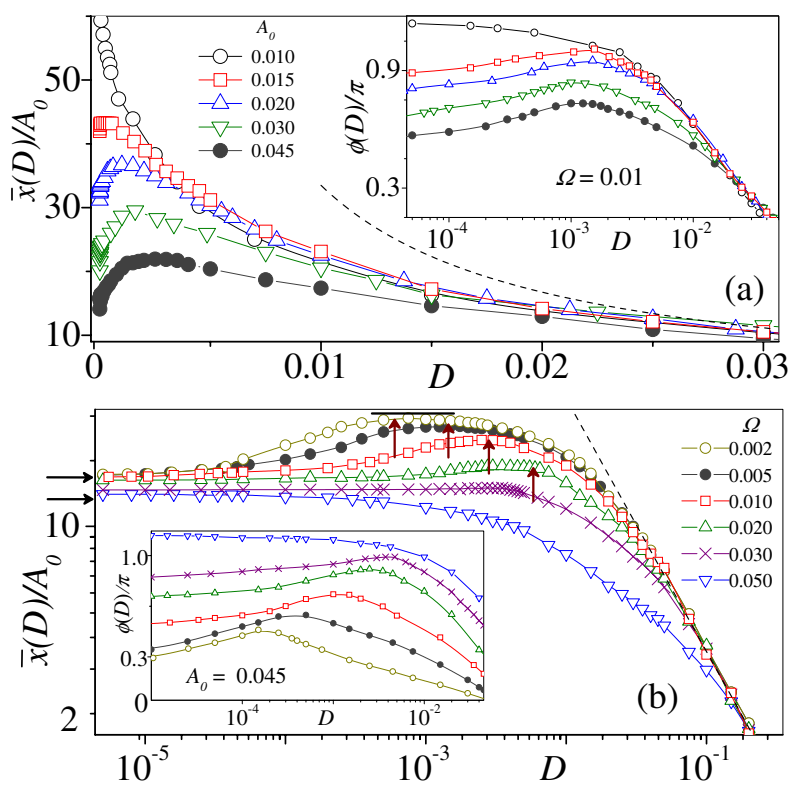

FIG. 2 (color online). Geometric stochastic resonance. (a) $\bar{x}(D)$ versus $D$ for different values of the ac drive parameters $A_{0}$ at $\Omega=0.01$ in (a) and $\Omega$ at $A_{0}=0.045$ in (b). Other parameters are: $x_{L}=y_{L}=1$ and $\Delta=0.1$. The dashed curve in (a) represents our predicted asymptotic decay $\bar{x} / A_{0}$ for $D \rightarrow$ $\infty$. In (b) we display our predictions for: the range of variability of $\bar{x}(0)$ (horizontal arrows), the SR peak position $D_{\max }$ (vertical arrows), the SR peak height $\bar{x}\left(D_{\max }\right)$ (top line), and the decay law $\bar{x}(D \rightarrow \infty)$ (dashed line); see text. the particle against the walls of the container opposite to the dividing wall, then the average particle displacement $\langle x(t)\rangle$ approaches a square waveform with amplitude $x_{L}$. Thus, the particle gets pushed against the container partition twice per cycle; if it goes through the opening, $\langle x(t)\rangle$ traces a symmetric, two-sided square wave; if it does not, its average displacement is restricted to an asymmetric, one-sided square wave, on either the positive or the negative side of the dividing wall. On taking the Fourier series of $\langle x(t)\rangle$ with period $2 \pi / \Omega$, the amplitude of its fundamental harmonic component turns out to be, respectively, $4 x_{L} / \pi$ for the two-sided waveform, and $2 x_{L} / \pi$ for the onesided waveform. These observations provide an upper bound to $\bar{x}$,

$$
\bar{x} \leq(4 / \pi) x_{L},
$$

and the onset condition for SR,

$$
A_{0} / \Omega \geq(4 / \pi) x_{L},
$$

$A_{0} / \Omega$ being the driven oscillation amplitude of an unconstrained Brownian particle. The SR peak, $\bar{x}\left(D_{\max }\right)$, approaches the upper bound in Eq. (2) at vanishingly low $\Omega$, see Fig. 2(b); whereas from Eq. (3), for our simulation parameters one obtains $A_{c}=0.013$ in Fig. 2(a) and $\Omega_{c}=$ 0.035 in Fig. 2(b). The consistency of these analytical results with the simulations is quite satisfactory.

At variance with SR in a bistable potential, the particle oscillations are not drastically suppressed in the zero-noise limit. An estimate for $\bar{x}(0) \equiv \lim _{D \rightarrow 0} \bar{x}(D)$ can be obtained by noticing that the probability for the particle to cross the pore and, therefore, to execute a full oscillation is $\Delta / y_{L}$, whereas the probability to get trapped on either side of the partition is $\left(1-\Delta / y_{L}\right)$. Accordingly,

$$
\bar{x}(0)=\left(x_{L} / 2\right)\left(1+\Delta / y_{L}\right) \kappa(\Omega),
$$

with $\kappa=4 / \pi$ for $\Omega \rightarrow 0$, see Eq. (2), and $\kappa=1$ for $\Omega \rightarrow$ $\Omega_{c}$. This defines a relatively narrow variability range for $\bar{x}(0)$ as a function of $\Omega$. Well above the onset threshold (3), namely, for $\Omega \ll \Omega_{c}$, for $D \rightarrow 0$ the curves in Fig. 2(b)

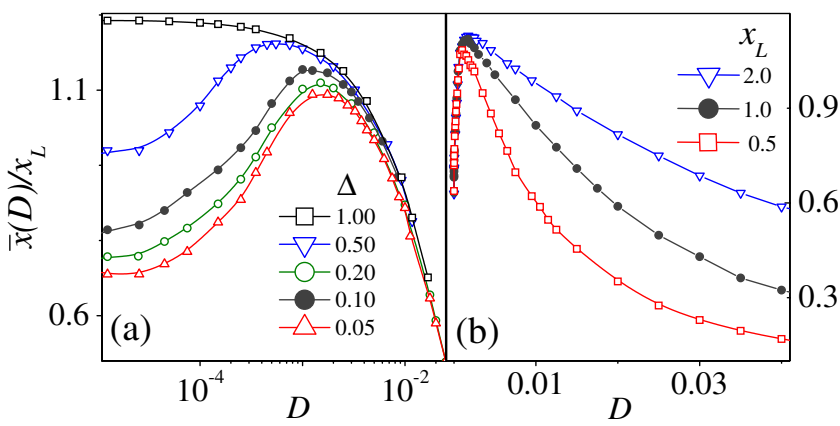

FIG. 3 (color online). Geometric dependence of this SR. $\bar{x}(D)$ versus $D$ for different values of $\Delta$ (a) and $x_{L}$ (b). Other parameters are: $y_{L}=1, A_{0}=0.045, \quad \Omega=0.005$ and (a) $x_{L}=1$, (b) $\Delta=0.1$. In both panels $\bar{x}(0)$ is very close to our prediction from Eq. (4) with $\kappa(\Omega)=4 / \pi$. 
clearly tend to the upper bound of $\bar{x}(0)$ in Eq. (4); correspondingly, the upper bound of noise amplification via SR is $\bar{x}(D) / \bar{x}(0) \leq 0.5$.

To check these predictions we modified the container geometry. In Fig. 3 the response amplitude $\bar{x}(D)$, in units of $x_{L}$, is plotted versus $D$ for different $\Delta$ 's, panel (a), and $x_{L}$ 's, panel (b). In agreement with Eq. (4), $\bar{x}(0) / x_{L}$ in panel (a) grows linearly with $\Delta$, while in panel (b) is seemingly insensitive to $x_{L}$. Note that $\bar{x}(0)$ approaches the upper bound (2) only for $\Delta \rightarrow 1$, thus implying that geometric SR happens for any finite pore width.

The dependence of the decaying tails of $\bar{x}(D)$ on $\Delta$ and $x_{L}$ is also consistent with our geometric interpretation. Indeed, the $D \rightarrow \infty$ behavior of $\bar{x}(D)$ can be analyzed in terms of the average time, $\tau_{1}(D)$, for an unbiased Brownian particle to diffuse across one compartment. Such a time constant is easily obtainable by analytical means [9], $\tau_{1}(D)=x_{L}^{2} / 3 D$. In the presence of strong noise, the geometric constriction exerted by the pore grows ineffective; diffusion along the $x$-axis is then described by an ac forced damped Brownian motion with effective damping constant $\tau_{1}^{-1}$, so that the corresponding $\Omega$ component of $\langle x(t)\rangle$ gets suppressed both in amplitude, $A_{0} \tau_{1}(D) / \sqrt{1+\left[\Omega \tau_{1}(D)\right]^{2}}$, and phase, $\phi(D)=$ $\arctan \left[\Omega \tau_{1}(D)\right]$. For $\Omega \ll \Omega_{c}$, this leads to the $\Omega$-independent estimate, $\bar{x}(D) \sim A_{0} \tau_{1}(D)$, plotted in both panels of Fig. 2 (dashed curves). Note that $\tau_{1}$ is independent of $\Delta$ and quadratic in $x_{L}$, so that for $D \rightarrow \infty$ the tails of the curves of Fig. 3(a) collapse onto one curve and those of Fig. 3(b) scale proportional to $x_{L}$.

The SR peaks of $\bar{x}(D)$ occur at a certain value of the noise strength, $D_{\max }$, see Fig. 2, which weakly depends on $A_{0}$ and grows with $\Omega$. This property distinguishes geometric SR from ordinary SR, as here the $x(t)$ transitions between stable states (cavities) are not regulated by an activation rate over an energetic Arrhenius barrier. In the absence of a drive, $A_{0}=0$, the relaxation is characterized by some exit time, $\tau(\Delta)$, which is inversely proportional to $D$. Optimal synchronization between pore crossings and external drive requires that the particle switches compartment twice during one $A(t)$ cycle [1], i.e.,

$$
\tau(\Delta)=\pi / \Omega \text {. }
$$

As a consequence, $D_{\max }$ is proportional to $\Omega$.

For a quantitative analysis, we propose to define $\tau(\Delta)$ as the mean time a Brownian particle, uniformly distributed in one compartment, first crosses the pore. This quantity has been numerically computed and plotted in Fig. 4(a) for $A_{0}=0$ and different container geometries. On inserting the corresponding numerical data for $\tau(\Delta)$ into Eq. (5), we predict the values of $D_{\max }$ marked in Fig. 2(b) by vertical arrows.

The dependence of $\tau(\Delta)$ on the parameters $\Delta$ and $x_{L}$ is also instructive. For $\Delta=y_{L}$ we recover the $1 \mathrm{D}$ limit, $\tau_{1}$, as it should. At very small pore cross-sections, $\tau(\Delta)$ develops a nonanalytical dependence on $\Delta$, no matter what the compartment aspect ratio $x_{L} / y_{L}$. However, the ratio $x_{L} / y_{L}$ controls the interplay between diffusion along the $x$ and $y$ axis. For long compartments, $x_{L} / y_{L} \gg 1$, the exit time is of the order of $\tau_{1}$ until quite low $\Delta$, whereas for narrow compartments, $x_{L} / y_{L} \ll 1$, the exit time is dominated by the vertical diffusion of the particle towards the midsection of the cavity, where the pore is located, i.e., $\tau(\Delta) \sim\left(y_{L}-\Delta\right)^{2} / 12 D$ for $\Delta$ not too close to 0 and 1 [also shown in Fig. 4(a)]. To this regard, we remark that such a dependence of the exit time on $x_{L} / y_{L}$ is not inconsistent per se with a reduced 1D description of the Brownian diffusion along the $x$ axis. However, none of the assumptions introduced in Ref. [4] to accommodate for SR in the framework of the Fick-Jacobs kinetics [11], apply to the present case. How to possibly define an entropic barrier corresponding to a structureless partition hole and, therefore, how to interpret our results within the formalism of Ref. [4] is the subject of an ongoing investigation. In particular, it should be explained why the curves in Fig. 3(b) do not show a significant dependence on the cavity volume, being the entropy of a Brownian particle proportional to the logarithm of the volume accessible to it $[4,11]$. In fact, the SR mechanism reported here appears to be a $2 \mathrm{D}$ geometric effect irreducible to a $1 \mathrm{D}$ problem with
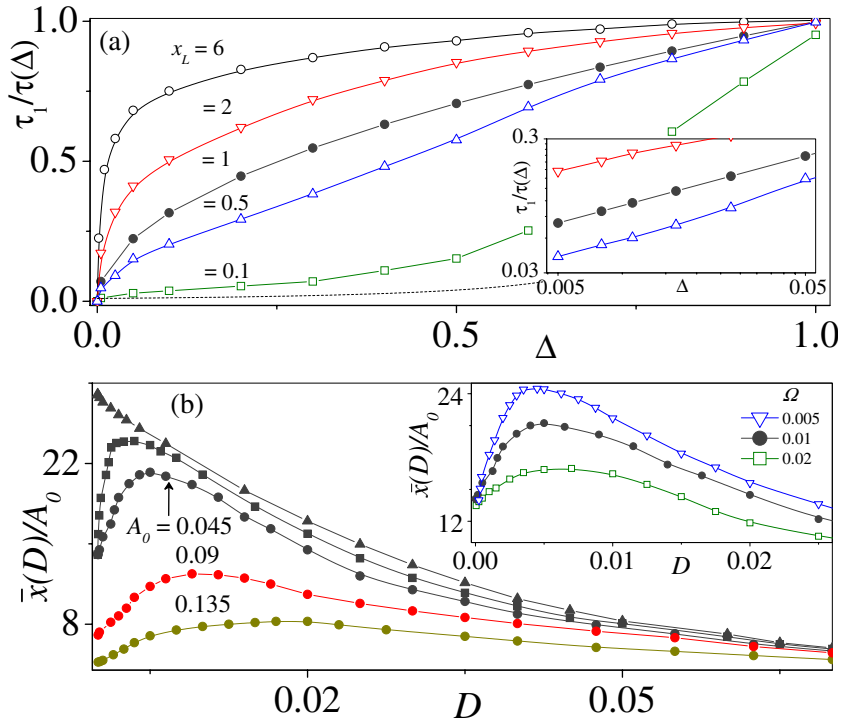

FIG. 4 (color online). Dependence of SR on the pore geometry. (a) $\tau(\Delta)$ in units of $\tau_{1}$, for zero drive, cavities of different lengths, and uniform initial particle distribution. The dashed curve represents the vertical diffusion time, $\left(y_{L}-\Delta\right)^{2} / 12 D$ (see text), for the shortest cavity. Inset: log-log plot of $\tau_{1} / \tau(\Delta)$ vs $\Delta / y_{L}$ for $\Delta \rightarrow 0$. (b) $\bar{x}(D)$ versus $D$ for different pore geometries: spout (circles), hole (squares), and funnel (triangles). $A(t)$ is a square waveform with $\Omega=0.01$ and amplitude as in the legend. Inset: $\bar{x}(D)$ versus $D$ for a spoutlike pore at $A_{0}=0.045$ and different $\Omega$. Other simulation parameters are: $x_{L}=y_{L}=1, \Delta=0.1$, and $x_{0}=0.1$ (where it applies). 
an entropic potential, so that the term geometric $S R$ would be more appropriate for our case.

The phase delay, $\phi(D)$, of the fundamental harmonic component of $\langle x(t)\rangle$ with respect to $A(t)$ is plotted in the insets of Fig. 2. By comparing the corresponding curves for $\bar{x}(D)$ and $\phi(D)$, we notice that: (i) $\phi(D)$ also exhibit SR-like peaks, which shift to higher $D$ on increasing $\Omega$ [Fig. 2(b)], while being almost insensitive to $A_{0}$ [Fig. 2(a)]. Note that in a two-state model $\phi(D)$ would be a monotonically decreasing function of $D$ [1]. (ii) The phase peaks are suppressed at low $\Omega$, where the SR signature of $\bar{x}(D)$ is most prominent; (iii) In the zero-noise limit, in contrast to $\bar{x}(0), \phi(0)$ strongly depends on $\Omega$ and $A_{0}$; for $D \rightarrow \infty$ the expected asymptotic behavior, $\phi(D) \sim \Omega \tau_{1}(D)$, was recovered (not shown). The nonmonotonic behavior of $\phi(D)$ signals the appearance of two-sided oscillating trajectories in the averaging ensemble of $\langle x(t)\rangle$, with the particle crossing more and more frequently the pore in unison with $A(t)$. According to our interpretation of the phenomenon under study, phase peaks are natural SR precursors. However, if the SR onset condition (iii) is not met, $\phi(D)$ tends to $\pi / 2$, as expected, as the particle is restricted to oscillate inside one cavity most of the time [1].

In real experiments at small-length scales, the geometry of the partition wall and its opening(s) are often not fully controllable. In Fig. 4(b) we plot $\bar{x}(D)$ versus $D$ for the three different pore shapes sketched in Fig. 1(b). In order to enhance geometric effects, we used a square waveform $A(t)$ also with amplitude $A_{0}$ and angular frequency $\Omega$. Moreover, the pore width, $x_{0}$, was taken not too small, lest the different pore geometries become indistinguishable. Funnel-like pores tend to channel the Brownian trajectories through the opening, no matter what their cross section; thus, no SR evidence was detected, as anticipated in Ref. [4]. On the contrary, spoutlike pores exhibit enhanced SR peaks and a distinct dependence on the cavity geometry. Indeed, under the pressure exerted by the drive, the exit time through a spout of width $x_{0}$ is of Arrhenius type, namely, a function of the pore cross section, proportional to $e^{A_{0} x_{0} / D}$. Owing to the SR condition (5), $D_{\max }$ is now expected to grow almost linearly with $A_{0}$ and logarithmically decreases with $\Omega$, in qualitative agreement with our simulations. Note that, as soon as the pore shape comes into play, i.e., for $x_{0} A_{0} \gg D$, the $A_{0}$ dependence of the exit time cannot be ignored any more and a quantitative analysis of these results requires going beyond the approximations of linear response theory [1]. In the opposite limit, the pore can be well modeled by a simple hole, as we initially did.

We expect that geometric SR can be best demonstrated in vortex superconducting devices [12]. This class of artificial devices is presently attracting growing interest be- cause of potential applications to flux qubits, SQUIDs and superconducting rf filters. Superconducting samples with two vortex boxes connected by a thin pore of almost any geometry can be fabricated. Vortices are trapped inside the boxes with binding energy of the order of $\Phi_{0}^{2} L_{t} / \lambda^{2}$, where $\Phi_{0}$ is the magnetic flux quantum, $\lambda$ is the London penetration depth, and $L_{t}$ is the depth of the two vortex traps. The vortex density $n=H / \Phi_{0}$, is controlled by the intensity $H$ of the applied magnetic field. In the dilute limit, $H \lesssim \Phi_{0} / \lambda^{2}$, the vortex-vortex interactions become negligible, so that the transport properties of a single trapped vortex are not overshadowed by many-body effects. ac drives and noise sources can be easily implemented as Lorentz forces generated by independent electric currents injected into the sample parallel and perpendicular to the pore axis. Detection of SR under such experimental conditions is only regulated by the applied current sources; in particular, the noise parameter $D$ can be varied independently of the constant sample temperature.

We thank the RIKEN RICC for providing computing resources. We acknowledge partial support from the NSA, LPS, ARO, NSF Grant No. 0726909, the EPSRC Grants No. EP/D072581/1 and No. EP/F005482/1, and the AQDJJ.

[1] L. Gammaitoni et al., Rev. Mod. Phys. 70, 223 (1998); for an update see L. Gammaitoni et al., Special issue on Stochastic Resonance [Eur. Phys. J. B 69, 1 (2009)].

[2] D. Reguera et al., Phys. Rev. Lett. 96, 130603 (2006).

[3] B. Hille, Ion Channels of Excitable Membranes (Sinauer, Sunderland, 2001); P. Hänggi and F. Marchesoni, Rev. Mod. Phys. 81, 387 (2009).

[4] P. S. Burada et al., Phys. Rev. Lett. 101, 130602 (2008); P. S. Burada et al., Eur. Phys. J. B 69, 11 (2009).

[5] R. Zwanzig, J. Phys. Chem. 96, 3926 (1992); W. Sung and P. J. Park, Phys. Rev. Lett. 77, 783 (1996).

[6] see, e.g., P. S. Burada et al., Chem. Phys. Chem. 10, 45 (2009).

[7] M. Borromeo et al., Eur. Phys. J. B 69, 23 (2009).

[8] P. Kloeden and E. Platen, Numerical Solutions of Stochastic Differential Equations (Springer, Berlin, 1999).

[9] C. W. Gardiner, Handbook of Stochastic Methods (Springer, Berlin, 2004).

[10] E. Heinsalu et al., Eur. Phys. J. B 69, 19 (2009).

[11] M. H. Jacobs, Diffusion Processes (Springer, New York, 1967).

[12] J. E. Villegas et al., Science 302, 1188 (2003); Y. Togawa et al., Phys. Rev. Lett. 95, 087002 (2005); S. Savel'ev and F. Nori, Nature Mater. 1, 179 (2002); D. Cole et al., Nature Mater. 5, 305 (2006); S. Ooi et al., Phys. Rev. Lett. 99, 207003 (2007). 